\title{
CONVOCATORIAS DE ARTE, LA PRESENTACIÓN UTÓPICA DE UN FALLO EN EL SISTEMA
}

\author{
Sandra Villar Amigo
}

Investigadora independiente

\begin{abstract}
Resumen
Las convocatorias en el arte son requerimientos indispensables de obtención en el inicio de la carrera artística. La presencia de los artistas emergentes en este tipo de convocatorias ayuda a fortalecer su currículum de artista, a entrar en el circuito artístico y a ser interesante en el mundo del arte. Tan mínimo elemento, forma parte del proceso selectivo del arte y puede encauzar o derribar el comienzo de una carrera artista. Con el fin de analizar las diferentes partes de las convocatorias, se toma como un ejemplo concreto el concurso Ertibil y la provincia de Vizcaya que actuará de hilo conductor para abordar y reflexionar sobre los pros y los contras de estos controvertidos "apoyos a la creación de los artistas emergentes". Desde las bases y los resultados hasta la posibilidad de vivir de estas ayudas.
\end{abstract}

Palabras-clave: CONVOCATORIAS ARTÍSTICAS INSTITUCIONALES; ERTIBIL (CONVOCATORIA DIPUTACIÓN FORAL DE BIZKAIA); CÍRCULO DE ARTE; BECAS PARA ARTISTAS

\section{CALLS FOR ART, THE UTOPIAN PRESENTATION OF A MISTAKE IN THE SYSTEM}

\section{Abstract}

The calls in the art are indispensable requirements of procurance at the beginning of the artistic career. The presence of emerging artists in this type of convocations helps to strengthen their artist resume, to enter the artistic circuit and to be interesting in the world of art. As minimal element, it is part of the selective process of art and can get into or bring down the beginning of an artist career. In order to analyze the different parts of the calls, it is taken as a concrete example of Ertibil grant and of the province Vizcaya, which will act as a guide to approach and discuss the pros and cons of these controversial "supports for the creation of the emerging artists". From the bases and the results to the possibility of living from these grants.

Keywords: ARTISTIC INSTITUTIONAL CALLS; ERTIBIL (PROVINCIAL COUNCIL OF BIZKAIA CALL); CIRCLE OF ART; GRANTS FOR ARTISTS

\footnotetext{
Villar Amigo, Sandra. "Convocatorias de arte, la presentación utópica de un fal7o en el sistema“. AusArt 5(2): 55-67. DOI: 10.1387/ausart.18167
}

\section{AUSART}




\section{LAS CONVOCATORIAS}

'Convocatoria' es un término general con el que se designa al llamamiento que invita u obliga a participar en algún evento o lugar. Cuando hablamos de convocatorias de arte nos referimos a ayudas, becas, concursos o premios en los que se valora una obra, proyecto artístico o la propia trayectoria del artista. Cada convocatoria tiene sus propias modalidades. Las ayudas o becas son destinadas a diversos ámbitos como la formación artística, la producción o creación, la residencia, la exhibición, la publicación, etc. En el caso de los premios o concursos dependen de una disciplina (pintura, escultura), un motivo (tema o elemento de referencia) o un colectivo al que desean reconocer su labor (comisarios, artistas emergentes).

Mi experiencia con las convocatorias de arte es como gestora cultural al colaborar en su organización ${ }^{1}$, como artista al aplicar a ellas y como investigadora, aunque esta última función resulta estar intrínseca en el término artista. Y es que para acceder y conseguir la selección en una convocatoria parece que antes debieras estudiar esta: su recorrido a lo largo de los años, disciplinas aceptadas, tendencias escogidas en ediciones anteriores y al actual jurado. Además del estudio, presentarse a una convocatoria "implica currarse un proyecto, un dossier, adecuar la propuesta a unas normas, cotejar documentos, horas y horas con un papeleo que, si no ganamos, nadie nos va a pagar" (Salanova 2017).

De todo el proceso, es la investigación previa a la presentación a la convocatoria de arte la que se encuentra invisibilizada. Un esfuerzo y metodología propios de un grupo de investigación que no ha sido reconocido. Por dichos motivos, me planteé elaborar un estudio sobre este trabajo. Debido a mi condición de estudiante de Máster el análisis tomaría la forma de un $\mathrm{tfm}^{2}$ que nunca se llegaría a presentar, dicho sea de paso. Como la facultad se localiza en Vizcaya y este ámbito geográfico era el que más conocía a nivel artístico hasta el momento, decidí explorar lo relacionado con el concurso Ertibil. 


\section{LA CONVOCATORIA COMO EL PROCESO SELECTIVO DEL ARTE. ESTUDIO DE CASO: ERTIBIL}

Ertibil es un consolidado concurso de ámbito de provincial, organizado por la Diputación Foral de Vizcaya. Cuenta con una dilatada trayectoria y ha sabido adaptarse a los tiempos desde su nacimiento en 1983. Se creó con el objetivo de apoyar a las iniciativas creativas de los artistas emergentes de Vizcaya, difundir su obra y promocionar las artes visuales en este territorio.

El primer paso de la metodología fue el de búsqueda, documentación y archivo, para la cual se contó con la inestimable ayuda de dos personas cercanas al concurso. Por un lado, Aurelio Vega García, Técnico en dirección de proyectos culturales y responsable de la gestión de este concurso hasta aquel momento ${ }^{3}$. Por otro, Xabier Sáenz de Gorbea, a quien se podría definir como 'la voz del arte en el País Vasco' (Redondo 2017). Pero en cuanto a la investigación atañía, era su condición de 'coleccionista' de archivos histórico-artísticos lo que me condujo hasta él. Sería en su biblioteca personal donde se encontraría el primer catálogo editado de esta convocatoria, perdido en el resto de archivos y que por un casual él había guardado como otros muchos catálogos de exposiciones del País Vasco.

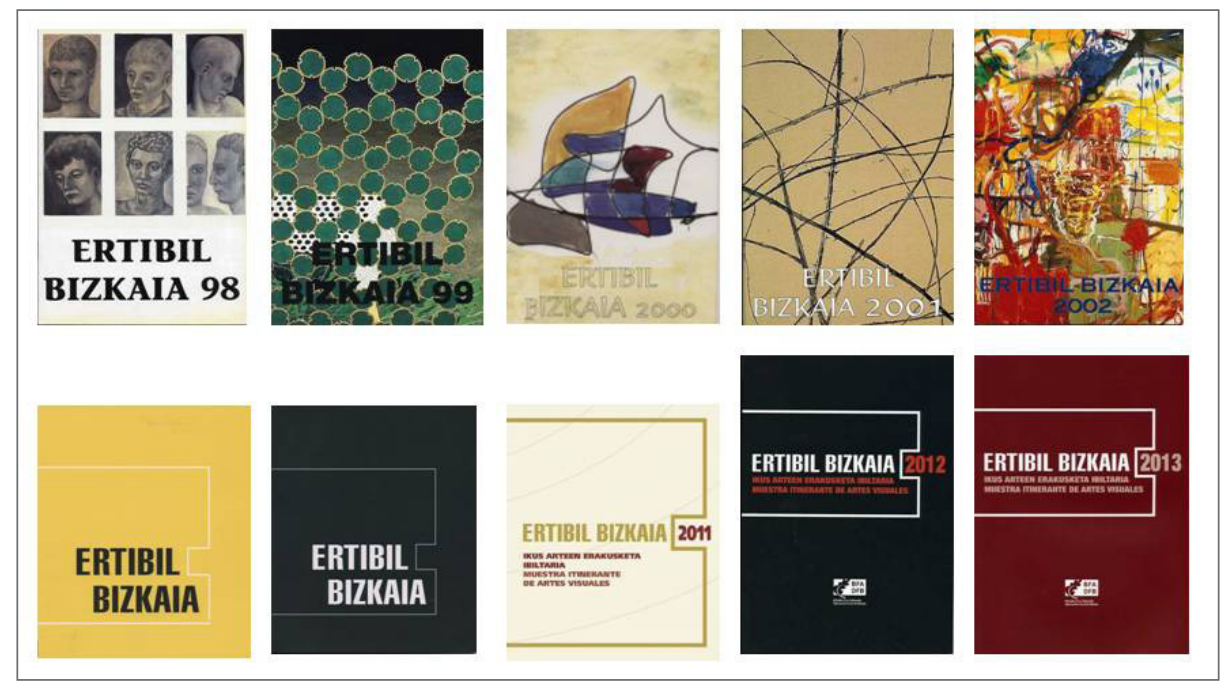

F1. Relación de portadas del Catálogo de Ertibil entre 1998-2002 y desde 2009-2013 
Gracias a las facilidades que ambos ofrecieron para la revisión de dicho material se pudo recabar todas las convocatorias y los catálogos desde la creación de este programa. Tras su catalogación, la creación de tablas de contenidos y su comparación, se vislumbraba una trayectoria de esta convocatoria hasta el año de su estudio 2014 y desde entonces no se ha dejado de analizar.

La evolución de los resultados, lejos de ser específica o singular, se mostró semejante a la progresión de otras convocatorias del panorama español. La exposición de las propiedades de este concurso en particular pone en evidencia las semejanzas y repeticiones de errores respecto a otros de índole similar. No se trata de generalizar un resultado concreto, sino de confrontar las bases y los resultados de diferentes convocatorias y meditar sobre su neutralidad y/o adecuación a los tiempos que corren.

Algunas de las características pueden resumirse en:

- Variación de las disciplinas artísticas aceptadas: ampliación del abanico de tipologías desde las iniciales: dibujo, pintura, escultura y grabado; hasta las más recientes: performance, instalaciones sonoras...

- Relación directa entre las disciplinas, materiales o procedimientos seleccionadas y los ofertados en la Facultad de Bellas Artes del País Vasco. A medida que evoluciona el tiempo se observa que hay nuevas especialidades que se ofrecen en la Universidad y que a partir de ese momento tienen presencia entre los seleccionados de dichas convocatorias. Un ejemplo bastante visible es la selección de piezas escultóricas o instalaciones realizadas en cerámica a partir de la implantación del Máster especializado en dicho material. Años atrás se mostraba una mayor preferencia por materiales como la madera, el hierro o incluso la escayola.

- Cuota destacable del jurado conformado por el profesorado de la Universidad de Bellas Artes de la Universidad del País Vasco UPVI EHU. Depende de las convocatorias podemos hablar de un profesor o incluso tres que forman parte del jurado. Una conclusión obvia es la posibilidad de reconocimiento de obra presentada y la dificultad para la realización de una valoración objetiva.

- Consonancia, coherencia o conexión entre las obras seleccionadas y gustos de los jurados. Esto se hace evidente por las diferencias de tendencia en la selección, así como el aumento de obras seleccionadas de cierta disciplina. Sin embargo, en la actualidad, es algo que se trata de evitar al premiar y seleccionar al menos una obra de 
cada 'conocimiento' o especialidad. Los cambios estéticos se observan incluso en las portadas de los catálogos (F1) de obras basadas en dibujo y pintura, a la valoración del diseño y la obra conceptual.

- Cantidades monetarias de los premios. Las cantidades han ido subiendo con los años y es digno de admiración si lo comparamos con premios de otras provincias. Solo el primer premio cede su obra al organizador del concurso -la Diputación de Bizkaia- y es el único que no podrá volver a presentarse a esta convocatoria. Además los seleccionados reciben una compensación económica, algo muy poco usual. Es un hecho muy singular que hace que más de uno desee la selección o el segundo y tercer premio, antes que el primero. Hay artistas que son seleccionados casi todos los años, algo que les supone un ingreso de $2000 €$ anuales. Compensa más la selección y recibir premios durante un par de años que la concesión del primer premio, ya no solo a nivel económico sino también curricular. Resulta un tanto irónico y parece contradecirse con las 'reglas del juego' que marcan los concursos, en los que se entiende que se participa buscando conseguir el primer premio.

- Muestra de los resultados del concurso en una exhibición, que recorre varias localidades de la provincia. Ha existido ligera variación en su trayectoria pero no se encuentra como dato de suma relevancia.

\section{LA INFLUENCIA E IMPORTANCIA DE LAS CONVOCATORIAS EN LOS CÍRCULOS DEL ARTE}

Tras realizar cursos con diferentes artistas reconocidos ${ }^{4}$ observo que a la pregunta de cómo hacer del arte tu profesión no hay una respuesta única. Sin embargo, sí que hay ciertos indicativos que pueden fortalecer la imagen del artista y entrar en el famoso 'círculo', que no tiene que ver con la calidad de la obra y otros conceptos quizá más importantes.

Conviene tener una serie de selecciones, premios, y en general, reconocimientos en convocatorias que sumados a la cercanía o presencia dentro de agrupaciones artísticas y/o ideológicas sirvan como aval para ser valorados como parte esencial de ese presente (por sus coetáneos) y como representantes de ese pasado (por sus correspondientes futuros). 
Una de las fórmulas del proceso que consolidan a los artistas comienza por la realización de exposiciones para posteriormente obtener selecciones o premios en convocatorias, que resultan de gran ayuda a la hora de conseguir becas y ayudas las cuales les permite producir más obra con la que desarrollar nuevas exposiciones. A su vez entre estos pasos habría que incluir estudios de especialización, cursos de artista y muchas otras actividades que hagan interesante tu biografía.

Pueden existir variaciones de orden, incluso hay casos en los que se gana un premio sin contar con un currículum previo, pero hay ciertos pasos que parecen tener que repetirse para ser valorado. El proceso se convierte en un círculo vicioso, una pescadilla que se muerde la cola, una ironía elitista y hermética. Y a pesar de lo duro que resulta entrar en esta rueda considero que es más sencillo entrar que mantenerse o salir de ella.

$Y$ es que en los tiempos que corren, con un bajo nivel de mecenazgo ${ }^{5}$ y sin demasiadas facilidades económicas para el desarrollo de la cultura, parece que una gran mayoría de los artistas aspiran a vivir de ayudas, a ser 'artistas de premios'. Éste es el término fue otorgado por Javier Díaz-Guardiola (2017) para definir a una serie de artistas que se dedican a enlazar diferentes becas y ayudas para poder vivir del arte. En su artículo, de manera abierta y sin tapujos, habla de una repetición de artistas que parecen ser omnipresentes (que son mostrados en dos exposiciones simultáneamente y en la misma ciudad o que reciben dos ayudas diferentes para un mismo proyecto artístico). Casos así, hacen plantearse si las elecciones se centran en las obras o más bien en los nombres. Si fuese lo segundo, el término 'fallo', comúnmente usado para designar el resultado de un concurso, le haría justicia.

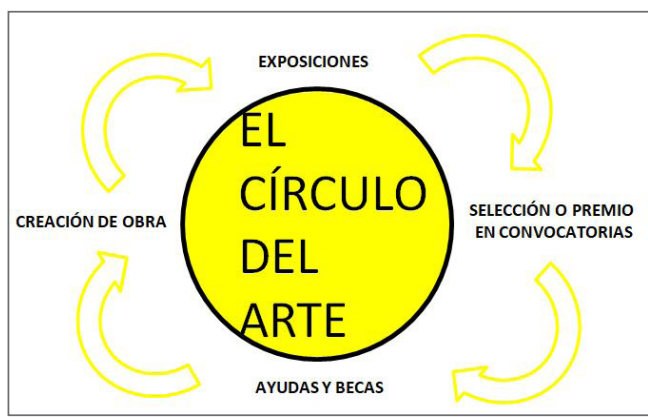

F.2 Representación del proceso para vivir de becas. (Creación propia)
Pero si nos ponemos en la situación del artista ¿quién rechazaría un premio, una ayuda, una selección con todo lo que significa? Si nos remitimos al caso de estudio de Ertibil, una selección supone: una exposición itinerante, un catálogo, un comentario de obra y menciones en artículos periodísticos. Así mismo, cualquier concurso o muestra te visualiza ante galeristas locales, compañeros y otros profesionales. 
Ahora bien, más allá de todo el 'prestigio', y aunque nunca se vaya a reconocer, lo que importa es que un premio en un concurso ofrece cierta cantidad económica y eso es lo que de verdad te permite seguir produciendo o simplemente comer. En el caso de Ertibil, se otorga ayuda económica tanto a los premiados como a los seleccionados y insisto en que es una característica muy positiva y por desgracia muy anómala en el resto de convocatorias.

Tras lo expuesto, no es difícil deducir que los premios y selecciones, o bien, se otorgan a personas que ya han tenido cierto reconocimiento en el mundo artístico, o bien, que la selección se lo concede. Habría que reflexionar sobre si estos concursos son una oportunidad real para los que están empezando o si son la concesión arbitraria de un cheque en blanco a los ya conocidos.

En el caso opuesto al de los artistas que disfrutan de varias ayudas, nos encontramos el más habitual y poblado, desgraciadamente, los artistas y licenciados en Bellas Artes que dejan el arte tras haberse presentado continuadamente a convocatorias y no haber obtenido resultado alguno. Una gran parte desisten sintiéndose defraudados por el sistema y lo que es peor, decepcionados y frustrados al poner en duda sus propias habilidades. Estas situaciones visualizan el lado más perverso del sistema, no muy lejano a la estructura socioeconómico piramidal y elitista, que habitualmente criticamos pero, que parecemos repetir sin preocupación.

A su vez, Guardiola nos habla de la existencia de circuitos del arte, en concreto de "la Santa Trinidad de las ayudas a artistas multidisciplinares de España", entre los que incluye Circuitos, Generaciones e Itinerarios. Además tiene presente la convocatoria del Banco BBVA (con una mejor cantidad monetaria) y las becas Injuve. Lo cierto es que si preguntásemos a cualquier experto en arte confirmará la anterior agrupación, quizá sin aludir a términos religiosos o divinos, pero sí lo acentuaría de otra manera. Seguramente dichos supuestos expertos también comentarían que existen varios circuitos de concursos o ayudas que se convierten en requisitos necesarios para la valoración de artistas en niveles más altos.

Podemos corroborar la existencia de estos circuitos, volviendo al concurso de estudio y el lugar geográfico que abarca. En Vizcaya existen ciertos puntos estratégicos para los artistas como: la Fundación BilbaoArte, las ayudas a la producción de la Diputación de Vizcaya y el Premio Ertibil. Pero en la última década se han sumado nuevas iniciativas impulsadas por la Facultad de Bellas Artes en colaboración con otras instituciones o empresas. Ejemplo de estas 
colaboraciones son: el Certamen de Arte Carlton, concurso de pintura en colaboración con el Hotel homónimo; Arteshop Bilbao, convocatoria de intervenciones en Comercios de la ciudad en colaboración con el Ayuntamiento de Bilbao y la Fundación BilbaoArte; además de otras muchas conferencias, cursos y actividades de formación complementaria que se vienen activando desde la Facultad. Todos ellos son circuitos al fin y al cabo, pero existe una gran diferencia y es que los programas fomentados por las universidades están pensados para estudiantes. Permiten que los alumnos puedan presentarse en condiciones similares y acaben la carrera con un pequeño portfolio.

Sin embargo en la redacción de los concursos hay muchas diferencias: "Hay concursos en los que las bases están redactadas de manera tal que compiten profesionales que juegan en ligas diferentes y no tiene sentido porque abarcan demasiados perfiles. Concursos públicos sin límite de edad, por ejemplo, cosa que en principio parece deseable y democrática, enfrentan a artistas o investigadores emergentes con profesionales de largo currículum e incluso profesores de universidad, con mucha más experiencia y recorrido vital en general" (Salanova 2017).

Cuando no existen iniciativas tales en las universidades, la salida al mercado del arte se hace más cuesta arriba de lo habitual y la posibilidad de conseguir una subvención con un currículum vacío se convierte en una utopía, incluso para los concursos para artistas emergentes. $Y$ es que en la actualidad, en las convocatorias de artistas emergentes no sorprende ningún nombre, las exigencias hacen que los artistas seleccionados tengan cierta trayectoria consolidada. De hecho es la misma Marisol Salanova (2017) quien habla de la importancia de este perfil de artistas con carreras impecables y que deben enmarcarse en tener la nacionalidad española, ser menor de 35 y tener el máximo número de estudios superiores, además claro de todos los méritos artísticos. Ella reivindica que este estándar de selección es una forma de asegurar el margen de beneficio que buscan las instituciones o empresas que convocan estas ayudas. Pero hay más fórmulas: también se pueden obtener ganancias cuando se ofrece una ayuda de producción y residencia. En estas ayudas, se subvenciona el material y se cede el sitio donde crearlo pero no se paga la mano de obra, que en términos artísticos serían los honorarios del artista. Al finalizar la beca una de las obras pasa a formar parte de la colección, con la gran ganancia que supone comprar la casa construida y pagar solo por el precio de los ladrillos. 


\section{EL FIN DE VIVIR DEL ARTE}

Hans Abbing ya comentó que "las artes no le habían ofrecido la alternativa romántica que había esperado. Ser artista supone trabajo duro y mal pagado" (citado en Pérez Ibáñez \& López-Aparicio 2016), y eso que en su caso pudo vivir del arte.

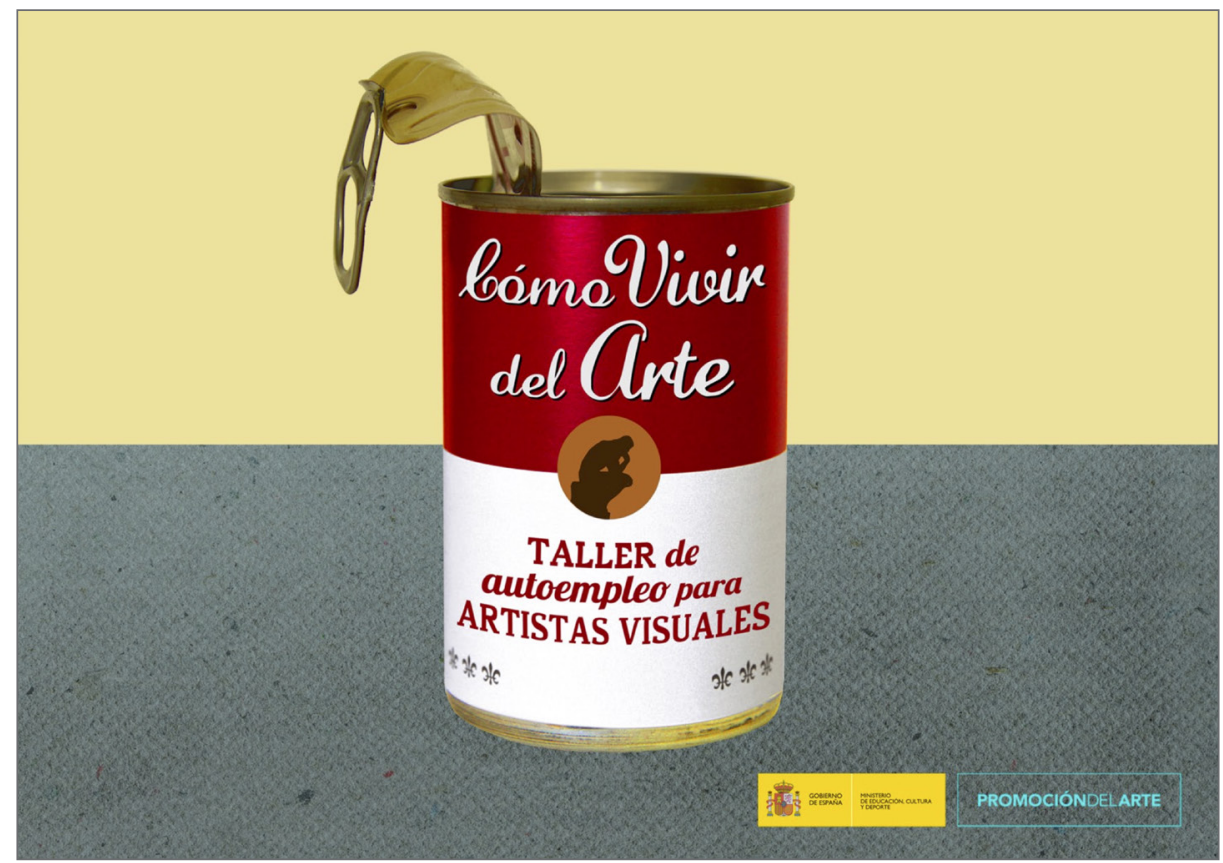

F.3 Portada del archivo online del Ministerio de Educación, Cultura y Deporte.

Los estudios de La actividad económica de los/las artistas en España (Pérez Ibáñez \& López-Aparicio 2016) confirman una difícil situación económica en el arte. La investigación ha analizado más de 1.100 encuestas realizadas a artistas que abordan cuestiones como la economía, la relación con el mercado y las variaciones desde el comienzo de la crisis, considerando el año 2008 el de inicio de ésta. Los datos son alarmantes: más del $80 \%$ de los artistas no tienen ni 5 años cotizados a la seguridad social, el $50 \%$ no llegan al salario mínimo interprofesional y tan solo el $15 \%$ de los encuestados puede vivir con los ingresos derivados de su trabajo como artista. Las opciones pasan por formar parte de los artistas de una galería de arte, trabajar junto con asociaciones y otras entidades con presupuestos públicos, obtener subvenciones o ayudas y sobre todo, trabajar en otros sectores. 
Seguramente en otros sectores puede resultar sorprendente, pero los resultados muestran que si quieres vivir del arte en este país parece que tienes que atenerte a recibir premios y selecciones que ejercerán una doble función: la de proporcionar ingresos para tus necesidades básicas y la de aval de confianza para posteriores ayudas.

Ante tal panorama, subyace una gran pregunta: cómo solventarlo. Pues bien, parece viable adoptar iniciativas similares a las promulgadas en estados cercanos. Carmen Oviedo Cueva defiende esta opción tras la revisión de los sistemas de comercialización y activación de la cultura adoptados por algunos estados de la Unión Europea con óptimos resultados. Su esclarecedor estudio (Oviedo Cueva 2014) concluye con una propuesta de medidas que mejorarían el panorama actual del arte, ahora solo faltarían ojos que lo leyesen, oídos que lo escuchasen y manos que finalmente lo aplicasen.

\section{LA NO CONCLUSIÓN}

Las convocatorias se crearon para promover y facilitar diferentes partes del proceso artístico desde la formación, la creación, la exhibición... Eran un apoyo para ayudar a la profesionalización y reconocimiento de diferentes colectivos y actividades relacionadas con el arte. En la actualidad, dichas convocatorias establecen un nuevo y frustrante estilo de vida en el que el artista sobrevive presentándose de manera continuada y sin interrupciones a cada convocatoria.

Para muchos, su producción y necesidades de vida básicas dependen directamente del resultado de estas ayudas, siendo en muchas ocasiones su único medio de ingresos. Para otros, su sumisión se debe a la obligatoriedad de reconocimiento artístico antes de cumplir los 35 años de vida, estimado de manera general como la edad máxima para ser artista emergente. A partir de ese momento, los artistas llegan 'considerados' como tal o quedan casi desligados del sistema del arte.

Unos y otros, viven dependientes de las distintas convocatorias de arte, que no son más que unas bases casi de copia y pega, cuyas vicisitudes generales se repiten tanto en ámbitos locales como en los nacionales. Son muchos los críticos y pensadores que de la misma manera, han analizado el sistema de 
becas, ayudas y convocatorias, opinando sin censurarse sobre los pros y los muchos contras que tienen. $Y$ aún así con las muchas mejoras que deben hacerse para ser justas y efectivas, la respuesta a esas convocatorias es masiva.

A la pregunta de ¿por qué?, seguramente la respuesta sería la necesidad. Estando en un tramo de edad que se define como lo mejor de la vida nos encontramos sin opciones: con una sociedad cada vez más dividida e individualizada, sin apenas oportunidades laborales en las que inscribirnos y con un embudo de consolidación artística lleno de demandantes. Desde mi punto de vista vivir de las convocatorias debería ser una elección, no la única alternativa ante la falta de trabajo y profesionalización de este nuestro sector.

A la pregunta de ¿para qué?, la respuesta sería para poder seguir en los circuitos del arte. Pero si faltan políticas que favorezcan el fortalecimiento del arte y en general de la cultura, para qué servirá la creación de una convocatoria y menos aún los esfuerzos por conseguir ser premiados en ella. Porque un placebo sirve de paliativo dando tiempo, hasta que el sujeto no pueda evitar enfrentarse con la realidad. Entonces cuál es el sentido tirarse años preparando la subida al Everest pensando en alcanzar la cima si al llegar solo espera el precipicio que dejó la propia cumbre desprendida por exceso de congelación. Cual iceberg de su glaciar, cual convocatoria sin un plan que lo consolide como parte de una estructura con futuro.

Si algo se pretendía con esta personal abstracción es provocar una reflexión con el conocimiento previo y los ejemplos que cada cual domine; considero que todos tenemos la responsabilidad de analizar las estructuras que crean nuestra realidad y reivindicar los cambios necesarios para que esto no suceda en un futuro cercano.

Pero claro, quién puede luchar por un futuro con el agotamiento que supone sobrevivir a nuestro presente.

\section{Referencias}

Aláez Vasconcellos, Irene, Belén Gil Jiménez, Gerardo Quintana Jiménez \& Patricia Romera. 2014. "Cómo vivir del arte: Taller de autoempleo para artistas visuales". Madrid: MECD. https://www.mecd.gob.es/cultura-mecd/dms/mecd/cultura-mecd/areas-cultura /promociondelarte/ComoVivirArteB.pdf 
Boltanski, Christian. 2016 "Christian Boltanski: 'Para un artista toda su vida es una forma de entender su trauma'” Entrevista por Belén Palanco. Exit Express, 27 jul. http://exit-express. com/christian-boltanski-para-un-artista-toda-su-vida-es-una-forma-de-entender-sutrauma/

Díaz-Guardiola, Javier. 2017. "'Circuitos y 'generaciones': De profesión, artista de premio". ABC Cultura, 8 feb. www.abc.es/cultura/cultural/abci-circuitos-y-generaciones-profesion-artista-premio-201702080122_noticia.html

Ertibil. 1986. Ertibil Bizkaia 86: Arte plastikoen erakusketa ibiltaria, katalogo [Exposición itinerante de artes plásticas, catálogo]. Bilbao: Bizkaiko Foru Aldundia

- 1987. Ertibil 87: Herriz herriko plastika-artezko erakusketa [Muestra itinerante de artes plásticas]. Bilbao: Bizkaiko Foru Aldundia

- 1988. Ertibil Bizkaia 88: Herriz herriko plastika-artezko erakusketa [Muestra itinerante de artes plásticas]. Bilbao: Bizkaiko Foru Aldundia

- 1989. Ertibil 89: Herriz herriko plastika-artezko erakusketa [Muestra itinerante de artes plásticas]. Bilbao: Bizkaiko Foru Aldundia

- 1990. Ertibil Bizkaia 1990: Herriz herriko plastika-artezko erakusketa [Muestra itinerante de artes plásticas]. Bilbao: Bizkaiko Foru Aldundia

- 1991. Ertibil Bizkaia 91: Herriz herriko plastika-artezko erakusketa [Muestra itinerante de artes plásticas]. Bilbao: Bizkaiko Foru Aldundia

- 1992. Ertibil Bizkaia 92: Herriz herriko plastika-artezko erakusketa [Muestra itinerante de artes plásticas]. Bilbao: Bizkaiko Foru Aldundia

- 1993. Ertibil Bizkaia 93: Herriz herriko plastika-artezko erakusketa [Muestra itinerante de artes plásticas]. Bilbao: Bizkaiko Foru Aldundia

- 1994. Ertibil Bizkaia 94: Herriz herriko plastika-artezko erakusketa [Muestra itinerante de artes plásticas]. Bilbao: Bizkaiko Foru Aldundia

- 1995. Ertibil Bizkaia 95: Herriz herriko plastika-artezko erakusketa [Muestra itinerante de artes plásticas]. Bilbao: Bizkaiko Foru Aldundia

- 1996. Ertibil Bizkaia 96: Herriz herriko plastika-artezko erakusketa [Muestra itinerante de artes plásticas]. Bilbao: Bizkaiko Foru Aldundia

- 1997. Ertibil Bizkaia 97. Bilbao: Bizkaiko Foru Aldundia

- 1998. Ertibil Bizkaia 98: Herriz herriko plastika-artezko erakusketa [Muestra itinerante de artes plásticas]. Bilbao: Bizkaiko Foru Aldundia

- 1999. Ertibil Bizkaia 99: Herriz herriko plastika-artezko erakusketa [Muestra itinerante de artes plásticas]. Bilbao: Bizkaiko Foru Aldundia

- 2000. Ertibil Bizkaia 2000: Herriz herriko plastika-artezko erakusketa [Muestra itinerante de artes plásticas]. Bilbao: Bizkaiko Foru Aldundia

- 2001. Ertibil Bizkaia 2001: Herriz herriko plastika-artezko erakusketa [Muestra itinerante de artes plásticas]. Bilbao: Bizkaiko Foru Aldundia

- 2002. Ertibil Bizkaia 2002: Herriz herriko plastika-artezko erakusketa [Muestra itinerante de artes plásticas]. Bilbao: Bizkaiko Foru Aldundia

- 2003. Ertibil Bizkaia 03: Arte gaztea [Arte jóven]. Bilbao: Bizkaiko Foru Aldundia

- 2004. Ertibil Bizkaia 04: Arte gaztea [Arte joven]. Bilbao: Bizkaiko Foru Aldundia

- 2005. Ertibil Bizkaia 05: Arte gaztea [Arte joven]. Bilbao: Bizkaiko Foru Aldundia

- 2006. Ertibil Bizkaia 06: Arte gaztea : [Arte joven]. Bilbao: Bizkaiko Foru Aldundia

- 2007. Ertibil Bizkaia 07: Arte gaztea : [Arte joven]. Bilbao: Bizkaiko Foru Aldundia

- 2008. Ertibil Bizkaia 08: Arte gaztea : [Arte joven]. Bilbao: Bizkaiko Foru Aldundia

- 2009. Ikus-Arteen erakusketa ibiltarirako XXVI edizioa [Muestra itinerante de Artes Plásticas XXVI edición]. Bilbao: Bizkaiko Foru Aldundia

- 2010. Ertibil Bizkaia 2010: Ikus-Arteen erakusketa ibiltaria [Muestra itinerante de Artes Visuales]. Bilbao: Bizkaiko Foru Aldundia

- 2011. Ertibil Bizkaia 2011: Ikus-Arteen erakusketa ibiltaria [Muestra itinerante de Artes Visuales]. Bilbao: Bizkaiko Foru Aldundia 
- 2012. Ertibil Bizkaia 2012: Ikus-Arteen erakusketa ibiltaria [Muestra itinerante de Artes Visuales]. Bilbao: Bizkaiko Foru Aldundia

- 2013. Ertibil Bizkaia 2013: Ikus-Arteen erakusketa ibiltaria [Muestra itinerante de Artes Visuales]. Bilbao: Bizkaiko Foru Aldundia

- 2014. Ertibil Bizkaia 2014: Ikus-Arteen erakusketa ibiltaria [Muestra itinerante de Artes Visuales]. Bilbao: Bizkaiko Foru Aldundia

- 2015. Ertibil Bizkaia 2015: Ikus-Arteen erakusketa ibiltaria [Muestra itinerante de Artes Visuales]. Bilbao: Bizkaiko Foru Aldundia

- 2016. Ertibil Bizkaia 2016: Ikus-Arteen erakusketa ibiltaria [Muestra itinerante de Artes Visuales]. Bilbao: Bizkaiko Foru Aldundia

- 2017. Ertibil Bizkaia 2017: Ikus-Arteen erakusketa ibiltaria [Muestra itinerante de Artes Visuales]. Bilbao: Bizkaiko Foru Aldundia

McAndrew, Clare. 2017. El mercado español del arte en 2017. Barcelona: Fundación Bancaria La Caixa

Oviedo Cueva, Carmen. 2014. "Análisis comparativo de la situación laboral del artista en el marco europeo y propuestas de mejora para la implantación de un modelo nacional". Trabajo fin de máster, Univ. del País Vasco

Pérez lbáñez, Marta \& Isidro López-Aparicio. 2016. La actividad económica de los/las artistas en España: Estudio y análisis. Madrid: Fundación Antonio de Nebrija

Redondo Lara, Maite. 2017. “Xabier Sáenz de Gorbea ha sido la voz del arte vasco”. Deia, 3 marzo. http://www.deia.com/2017/03/03/ocio-y-cultura/cultura/xabier-saenz-de-gorbea-hasido-la-voz-del-arte-vasco

Salanova Burguera, Marisol. 2017. "Vivir de becas". Exit Express, 19 ene. http://exit-express. com/vivir-de-becas/

Villardón, María. 2017. "Para vivir del arte en España primero hay que morirse. OK Diario, 12 ago. https://okdiario.com/economia/2017/08/12/vivir-del-arte-espana-primero-morirse-1236526

\section{Notas}

${ }^{1}$ XXVI Concurso Patrimonio Nacional de Pintura Infantil y Juvenil para Centros Escolares.

2 Trabajo de fin de máster.

${ }^{3}$ Actualmente ostenta este cargo Mikel Murillo Aurrekoetxea.

${ }^{4}$ Itziar Okariz, Cristina Lucas, Daniel García Andújar, Lara Almarcegui y Concha Jerez.

${ }^{5}$ Fuentes: Cultura y mecenazgo, https://www.mecd.gob.es/cultura/areas/industriasculturales/ mc/cultura-mecenazgo/inicio.html, y Anuario de estadísticas culturales https://www.mecd. gob.es/servicios-al-ciudadano/estadisticas/cultura/mc/naec/portada.html, ambos en la web del Ministerio de Educación, Cultura y Deporte. [Último acceso: 14 de octubre de 2017].

\footnotetext{
(Artículo recibido 20-11-2017; aceptado 12-12-2017)
} 